\title{
NOTE ON BLOCKS OF SYMMETRIC GROUPS
}

\author{
TADASI NAKAYAMA and MASARU OSIMA
}

A conjecture concerning characterization of blocks of representations of symmetric groups by means of Young diagrams, given formerly by one of the writers, ${ }^{1}$ was affirmed recently by R. Brauer and G. de B. Robinson jointly.:) Namely, it was proved in Brauer's paper, relying on his general, and profound, theory of blocks in finite groups, that the number of $p$-blocks in a symmetric group $\Im_{n}$, of degree $n$, is exactly equal to the number of $p$-cores, or $p$-kernels in the terminology of NI, with $n-l p(l=0,1,2, \ldots)$ nodes, ${ }^{3)}$ and that certain statements designated as (A), (B) and (C) would secure that two irreducible representations with a same $p$-core belong to a same block (whence the conjecture itself when combined with the estimate of the number of blocks). Then these statements (A), (B), (C) were proved actually in Robinson's paper.

On the other hand the present writers had been able to prove, independently of Brauer and Robinson, first that the number of blocks is at least equal to the number given in the conjecture, and stated above, and then that representations in a same block have necessarily a same p-core. ${ }^{4)}$ It seems to the writers that these results, which they want to give in the following, supplement those of Brauer and Robinson, and help to clarify the inner structure of the matter. Indeed, the last statement forms the counter-part of the latter half of the Toronto proof and gives a second proof of the conjecture when combined with the former half, that is, with the above exact estimate of the number of blocks given in Brauer's papers, ${ }^{5}$ in which lies however a very difficult portion of the matter. Even the first weak assertion may be used to take place of the exact estimate in the Toronto proof, though rather strainedly.

$\S 1$. Let $T$ be a diagram of $n$ letters, or nodes;

Received Sept. 4, 1950.

1) T. Nakayama, On some modular properties of irreducible representations of symmetric groups I, II, Jap. J. Math. 17 (1941); we refer to these papers as NI and NII.

2) R. Brauer, On a conjecture by Nakayama; G. de B. Robinson, On a conjecture by Nakayama, Trans. Roy. Soc. Canada, 41 (1947).

3) Terms core and node, in place of kernel and letter, are after Robinson.

4) The first weak assertion was shown by the former of the writers and the second was proved by the latter writer by combining his detailed structural analysis of diagrams with the former's argument for the first assertion.

5) Or, combined with Brauer's theory of blocks directly; see the end of the present note. 


$$
T=\left[\alpha_{i}\right]: \quad \alpha_{1} \supseteqq \alpha_{2} \supseteqq \ldots \supseteqq \alpha_{h} \quad\left(\sum \alpha_{i}=n\right),
$$

$\alpha_{i}$ being the length of its $i$-th row. Denote the length of its $j$-th column by $\gamma_{j}\left(j=1,2, \ldots, k\left(k=\alpha_{1}\right) ; \sum \gamma_{j}=n\right)$. Put further, as in NI,

$$
\beta_{i}=\alpha_{i}+h-i, \quad \delta_{j}=\gamma_{j}+k-j .
$$

Then evidently $\beta_{1}>\ldots>\beta_{h}>0, \delta_{1}>\ldots>\delta_{k}>0$. We refer to these numbers as $\alpha-, \beta-, \gamma-$, and $\delta$-numbers of the diagram $T$. The degree $f=f(T)$ of the irreducible representation of the symmetric group $\varsigma_{n}$ belonging to our diagram is given by $f=n ! \prod_{s<t}\left(\beta_{s}-\beta_{t}\right) / \Pi \beta_{i} !$.

Suppose now that $T$ has a hook ${ }^{6)} H$ of length $g$ (or $g$-hook briefly) at $(i, j)$; $g=\alpha_{i}+\gamma_{j}-i-j+1$. Put

$$
w=\gamma_{j}, \quad r=w-i+1 ;
$$

$r$ being the height of the hook. Let $T_{0}=T-H$ be the diagram of $n-g$ nodes obtained by removing the hook $H$ from $T$, and $f_{0}=f\left(T_{0}\right)$ be the degree of the corresponding irreducible representation of $\varsigma_{n-g}$. It was shown in NI that then

$$
\begin{aligned}
& f / f_{0}=\frac{n !}{(n-g) ! g !} K L M \text { with } K=\prod_{s<j}\left(\left(\delta_{s}-\delta_{j}\right) /\left(g+\delta_{s}-\delta_{j}\right)\right), \\
& L=\prod_{s<i}\left(\left(\beta_{s}-\beta_{i}\right) /\left(g+\beta_{s}-\beta_{i}\right)\right), \quad M=\prod_{i<s \cong r o}\left(\left(\beta_{i}-\beta_{s}\right) /\left(g-\beta_{i}+\beta_{s}\right)\right) .
\end{aligned}
$$

For the following is essential also the recurrence rule. ${ }^{7)}$ Let $H_{1}, H_{2}, \ldots$ be the totality of $g$-hooks in the diagram $T$, and let $r_{\nu}$ be the height of $H_{\nu}$. If $P$ is an element of $\Im_{n}$ containing a cycle of length $g$, and if $P_{0}$ is the permutation of $n-g$ letters obtained from $P$ by canceling this cycle, then

$$
\chi(T ; P)=(-1)^{r_{1}-1} \chi\left(T-H_{1} ; P_{0}\right)+(-1)^{r_{2}-1} \chi\left(T-H_{2} ; P_{0}\right)+\ldots
$$

where $\chi(T), \chi\left(T-H_{\nu}\right)$ denote the characters belonging to the diagrams $T, T-H_{\nu}$.

Now, a block of irreducible representations of $\mathfrak{S}_{n}$, with respect to a prime ${ }^{8)}$ $p$, is a class of irreducible representations which can mutually be connected by chains of irreducible representations such that neighboring two possess a common $(p-)$ modular component. $g(P) \chi(P) / f(\bmod p)$ characterize the block to which the character $\chi$ belongs, where $P$ runs in $\mathcal{S}_{n}, g(P)$ denotes the number of elements conjugate to $P$ in $\Im_{n}$, and $f=\chi(1)$ is the degree of the character,

6) For the notions of hooks and cores (kernels), and for their theory, see NI.

7) NI, Appendix. See also G. de B. Robinson, On the representations of the symmetric group, II, Amer. J. Math. 69 (1947); III, 70 (1948).

8) That $p$ is a prime we use only in congruences (and for the sake of our purpose). As to the structure of diagrams, $p$ need not be a prime in the following considerations. 
as beiore.

Consider a $p$-core $T_{0}$, i.e. a diagram without $p$-hook, consisting of $m=n-l p$ nodes. Let $T$ be the diagram of $n$ nodes obtained from $T_{0}$ by adding $I D$ nodes to its first row. Namely

$$
\begin{aligned}
& T=\left[\alpha_{1}, x_{2}, \ldots\right], \\
& T_{0}=\left[\alpha_{1}-l p, \alpha_{2}, \ldots\right], \alpha_{1}-l p \geqq \alpha_{2} \geqq \alpha_{3} \equiv \ldots
\end{aligned}
$$

The way to get $T_{n}$ from $T$ by removing $p$-hooks successively is unique. Hence, when $P$ is an element of $\Im_{n}$ possessing $l p$-cycles and when $P_{0}$ is obtained from $P$ by removing those $p$-cycles, then

$$
\chi(T ; P)=\chi\left(T_{0} ; P_{0}\right) \text {. }
$$

Suppose here that $P$ has exactly $l p$-cycles. Then the numbers of conjugates of $P, P_{0}$ are respectively $g(P)=\frac{n !}{l ! p^{l} c}, g\left(P_{0}\right)=\frac{(n-l p) !}{c}=\frac{m !}{c}$, where $c$ is a (common) factor which we are not interested in, and so $g(P) / g\left(P_{0}\right)=\frac{n !}{m ! l ! p^{l}}$.

On the other hand $f(T) / f\left(T_{0}\right)=\frac{n !}{m !(l p) !} K L M i$ with $K=\prod_{t<j}\left(\left(\delta_{t}-\delta_{j}\right) /(l p)\right.$ $\left.\left.+\delta_{t}-\delta_{j}\right)\right)\left(j=\alpha_{1}+1\right), L=M=1$. Here $K \equiv 1 \bmod p$ since $\delta_{t}-\delta_{j}$, the length of $(1, t)$-hook of $T$, is prime to $p$ for every $t=1,2, \ldots, \alpha_{1}$. Therefore

$$
\frac{g(P) \%(T ; P)}{f(T)} \equiv \frac{g\left(P_{0}\right) \%\left(T_{11} ; P_{t 1}\right)}{f\left(T_{0}\right)} \cdot \frac{l ! p^{l}}{(l p) !} \equiv \frac{g\left(P_{0}\right) \%\left(T_{0} ; P_{0}\right)}{f\left(T_{0}\right)}(-1)^{l} \bmod p .
$$

This congruence holds however a!so for those elcments $P$ which possess more than $l p$-cycles; for the both sides vanish then. Thus (3) is valid generally for $P$ with at least $l p$-cycles.

Let $\widetilde{T}_{0}$ be a second $p$-core with $\tilde{m}=n-\tilde{l} p$ nodes, say. Let $\tilde{T}$ be obtained from $\widetilde{T}_{0}$ by adding $\tilde{l} p$ nodes at the first row, just as $T$ is obtained from $T_{0}$. Then

$$
\frac{g(\tilde{P}) \chi(\widetilde{T} ; \widetilde{P})}{f(\widetilde{T})} \equiv \frac{g\left(\widetilde{P}_{0}\right) \chi\left(\widetilde{T}_{0} ; \widetilde{P}_{0}\right)}{f\left(\widetilde{T}_{0}\right)}(-1)^{\imath} \bmod p
$$

for every element $\widetilde{P}$ in $\Xi_{n}$ with at least $\tilde{l} p$-cycles, $\widetilde{P}_{0}$ being obtained from $\tilde{P}$ by canceling $\tilde{l} p$-cycles.

Now, suppose that the diagrams $T$ and $\widetilde{T}$ belong to a same block. We want to prove that then $T_{0}=\widetilde{T}_{0}$. Firstly $l=\tilde{l}$. For, if $l>\tilde{l}$ for instance, then for every $P=\widetilde{P}$ with exactly $l \dot{p}$-cycles (4) is congruent to $0 \bmod \dot{p}$, while (3) $\neq 0$ $\bmod p$ for a suitable choice of such $P$. Similarly $l<\tilde{l}$ can not be the case, and so $l=\tilde{l}$. Then we ought to have 


$$
\frac{g\left(P_{0}\right) \chi\left(T_{11} ; P_{0}\right)}{f\left(T_{0}\right)} \equiv \frac{g\left(P_{0}\right) \chi\left(\tilde{T}_{0} ; P_{0}\right)}{f\left(\tilde{T}_{0}\right)} \bmod p
$$

for every element $P_{0}$ of $\Im_{m}$. Then $T_{0}=\tilde{T}_{0}$ according to the case of highest blocks already analyzed formerly. ${ }^{91}$ We have

I. There are at least as many $p$-blocks of $\varsigma_{n}$ as there are $p$-cores with $n-l p$ nodes $(l=0,1,2, \ldots) . .^{\text {(0) }}$

§. In order to study the relationship of the characters of general diagrams and their cores, we have to make use of the following result obtained independently by Robinson ${ }^{11}$ and the latter of the writers. Namely:

The structure of a diagram $T=\left[\alpha_{i}\right]$ with respect to its p-hooks can be represented by a system $T^{*}$ of diagrams, at most $p$ in number; each node of (each component of) $T^{*}$ represents a p-hook (as well as its images) in $T$ and each g-hook in $T^{*}$ represents gp-hook in $T$ (and conversely every gp-hook in $T$ is associated with a g-hook in $\left.T^{*}\right)$.

That there are at most $p$ components in $T^{*}$ is closely related to, and in fact is derived from, the structure of diagrams with one $p$-hook studied in NII. ${ }^{12)}$ Indeed, the top left node of each component of $T^{*}$ is associated with a $p$-hook which may be added to the $p$-core $T_{0}$ of $T$. There are $p$ ways of such adjunction of a $p$-hook to $T_{0}$, and in fact one for each height $r=1,2, \ldots, p$ of the $p$-hook. We can thus designate each component of $T^{*}$ by the height $r$ of this $p$-hook, associated with its top left node. Allowing void diagrams as components, let $D^{(1)}, D^{(2)}, \ldots, D^{(p)}$ be $p$ components of $T^{*}$, where $D^{(r)}$, called the $r$-th component of $T^{*}$, belongs to the height $r$, and write $T^{*}=\left(D^{(1)}, D^{(2)}\right.$, $\left.\ldots, D^{(p)}\right)$. Let $D^{(r)}$ contain $l^{(r)}$ nodes; $l^{(r)}=0$ when $D^{(r)}$ is void. $l=\sum l^{(r)}$ is the number of nodes in $T^{*}$, and $n=m+l p$, where $m$ is the number of nodes in the core $T_{0}$.

It is also true that:

Conversely, if $T^{*}=\left(D^{(1)}, D^{(2)}, \ldots, D^{(p)}\right)$ is a system of $p$ diagrams, then there exists for each p-core $T_{0}$ a diagram $T$ whose structure concerning $p$-hooks is represented by $T^{*}$.

So we may summarize in:

ง) NI.

10) We give this assertion first, since it already has some bearing by itself, as was observed in the introduction, though rather weak, and moreover its proof may be regarded as a model of that of the stronger one in $\$ 2$.

11) Robinson, 1.c. 7) III.

12) NII, \&3. 
If $T_{0}$ is a p-core, diagrams $T$ with $T_{0}$ as p-core are in 1-1 correspondence with systems $\left(D^{(1)}, D^{(2)}, \ldots, D^{(p)}\right)$ of $p$ diagrams.

(The result may be applied to obtain a certain recurrence formula for the number of irreducible representations and that of self-conjugate irreducible representations of a symmetric group. Reserving this for a subsequent paper, we come back to our problem of blocks.)

Our system $T^{*}$ is a skew diagram in Robinson's sense. ${ }^{13)}$ The degree $f^{*}$ of the (reducible) representations of $S_{l}$ belonging to $T^{*}$, in his sense, is given by $f^{*}=\frac{l !}{l^{(1)} ! l^{(2)} ! \ldots l^{(p)} !} \bar{f}^{(1)} \bar{f}^{(2)} \ldots \bar{f}^{(p)}$, where each $\bar{f}^{(r)}$ denotes the degree of the irreducible representations of $\mathfrak{S}_{l(r)}$ belonging to $D^{(r)}$.

Consider a $g$-hook $H^{*}$ in $T^{*}$. If $H^{*}$ is in $D^{(r)}$, then $T_{0}^{*}=T^{*}-H^{*}=\left(D^{(1)}\right.$, $\left.D^{(2)}, \ldots, D^{(r)}-H^{*}, \ldots, D^{(p)}\right)$. The degree of the representation belonging to $T_{0}^{*}$ is $f_{0}^{*}=\frac{(l-g) !}{l^{(1)} ! \ldots\left(l^{(r)}-g\right) ! \ldots l^{(p)} !} \bar{f}^{(1)} \ldots \bar{f}_{0}^{(r)} \ldots \bar{f}^{(p)}$, where $\bar{f}_{0}^{(r)}$ is the degree of the representation belonging to $D_{0}^{(r)}=D^{(r)}-H^{*}$. Hence $f^{*} / f_{0}^{*}$ $=\frac{l !\left(l^{(r)}-g\right) ! \bar{f}^{(r)}}{(l-g) ! l^{(r)} ! \bar{f}_{0}^{(r)}}$. Here $\bar{f}^{(r)} / \bar{f}_{0}^{(r)}=\frac{l^{(r)} !}{\left(l^{(r)}-g\right) ! g !} \bar{K} \bar{L} \bar{M}$ by (1) in $\S 1$. So

$$
f^{*} / f_{0}^{*}=\frac{l !}{(l-g) ! g !} \bar{K} \bar{L} \bar{M} \text {. }
$$

This equation is the equivalent of (1) for the skew diagram $T^{*}$.

Taking away $l$ nodes from $T^{*}$ one by one, and observing that $\bar{M}$ of (5) is 1 in case $g=1$, we obtain, with factors $\bar{K}_{\lambda}, L_{\lambda}$ at each step,

$$
f^{*}=l ! \prod_{\lambda=1}^{l}\left(\overline{K_{\lambda}} L_{\lambda}\right) \text {. }
$$

If we remove from $T$ correspondingly $l p$-hooks $H_{1}, H_{2}, \ldots, H_{l}$ successively, then we reach the core $T_{0}$. Let $T_{l}=T, T_{l-1}=T_{l}-H_{1}, T_{l-2}=T_{l-1}-H_{2}, \ldots$, $T_{0}=T_{1}-H_{l}$ be the diagrams of successive stages. Denoting the degree of the irreducible representation belonging to $T_{\lambda}$ by $f_{\lambda}$, we have, by virtue of (1), $f_{l-\lambda+1} / f_{l-\lambda}=\frac{(n-(\lambda-1) p) !}{(n-\lambda p) ! p !} K_{\lambda} L_{\lambda} M_{\lambda}$ with $K_{\lambda}=\prod_{s<j_{\lambda}}\left(\left(\delta_{\lambda s}-\delta_{\lambda j_{\lambda}}\right) /\left(p+\delta_{\lambda s}-\delta_{\lambda j_{\lambda}}\right)\right)$, $L_{\lambda}=\prod_{s<i_{\lambda}}\left(\left(\beta_{\lambda s}-\beta_{\lambda i_{\lambda}}\right) /\left(p+\beta_{\lambda s}-\beta_{\lambda i_{\lambda}}\right)\right), M_{\lambda}=\prod_{i_{\lambda}<s \leqq w_{\lambda}}\left(\left(\beta_{\lambda i_{\lambda}}-\beta_{\lambda s}\right) /\left(p-\beta_{\lambda i_{\lambda}}+\beta_{\lambda s}\right)\right)$, where $\beta_{\lambda}, \delta_{\lambda}$ are the $\beta-, \delta$-numbers of $T_{l-\lambda+1},\left(i_{\lambda}, j_{\lambda}\right)$ is the position of $H_{\lambda}$ and $w_{\lambda}=r_{\lambda}+i_{\lambda}-1$ with the height $r_{\lambda}$ of $H_{\lambda}$. We have

$$
M_{\lambda}=\prod_{i_{\lambda}<\delta \leqq w_{\lambda}}(-1)=(-1)^{w_{\lambda}-i_{\lambda}}=(-1)^{r_{\lambda}-1} \bmod p \text {. }
$$

Hence

13) L.c. 7). A skew diagram is a diagram minus a top left partial diagram. 


$$
f / f_{0}=\prod_{\lambda=1}^{l}\left(f_{l-\lambda+1} / f_{l-\lambda}\right)=\frac{n !}{(n-l p) !(\phi !) !} \prod_{\lambda=1}^{l}\left(K_{\lambda} L_{\lambda} M_{\lambda}\right) .
$$

Denote (all) the nodes of $T^{*}$ corresponding to $H_{1}, H_{2}, \ldots, H_{l}$ by $H_{1}{ }^{*}, H_{2}{ }^{*}$, $\ldots, H_{l}{ }^{*}$. So the factors $\bar{K}_{\lambda}, \bar{L}_{\lambda}$ in (6) correspond to $H_{\lambda}{ }^{*}$. We have $\left.\left.\bar{K}_{\boldsymbol{s}<<j_{\lambda}, p \mid \delta_{\lambda s}-\delta_{\lambda j_{\lambda}}}=\boldsymbol{\delta}_{\lambda j_{\lambda}}\right) /\left(p+\delta_{\lambda s}-\delta_{\lambda j_{\lambda}}\right)\right)$. As $\left(\delta_{\lambda s}-\delta_{\lambda j_{\lambda}}\right) /\left(p+\delta_{\lambda s}-\delta_{\lambda j_{\lambda}}\right) \equiv 1 \bmod$

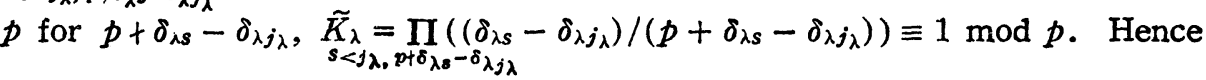

$$
K_{\lambda}=\overline{K_{\lambda}} \widetilde{K}_{\lambda}, \quad \widetilde{K}_{\lambda} \equiv 1 \bmod p .
$$

Similarly $L_{\lambda}=\bar{L}_{\lambda} \widetilde{L}_{\lambda}, \widetilde{L}_{\lambda} \equiv 1 \bmod p$, while $\prod_{\lambda} M_{\lambda} \equiv(-1)^{\left(r_{\lambda}-1\right)} \bmod p$. By (6) we have $f / f_{0}=\frac{n !}{(n-l p) !(p !)^{l}} \Pi\left(\bar{K}_{\lambda} L_{\lambda}\right) \Pi\left(\tilde{K}_{\lambda} \tilde{L}_{\lambda}\right) \Pi M_{\lambda}=\frac{n ! f^{*}}{(n-l p) ! l !(p !)^{l}}$ $\Pi\left(\widetilde{\bar{K}_{\lambda}} \widetilde{L_{\lambda}}\right) \Pi M_{\lambda}$, or

$$
f / f_{0}=\frac{n ! f^{*}}{m ! l !(p !)^{l}} \Pi\left(\widetilde{K}_{\lambda} \widetilde{L}_{\lambda}\right) \Pi M_{\lambda}
$$

with $\Pi\left(\tilde{K}_{\lambda} \tilde{L}_{\lambda}\right) \equiv 1, \Pi M_{\lambda} \equiv(-1)^{a} \bmod p$, where $q=\sum\left(r_{\lambda}-1\right)$.

(Denote the exponent of the (highest) power of $p$ dividing an integer $a$ by $e(a)$. As $T_{0}$ has no $p$-hook, we have $e\left(f_{0}\right)=e\left(f\left(T_{0}\right)\right)=e(m !)$. Since $e\left(l !(p !)^{l}\right)$ $=e((l p) !)$, we have further, from (8), the formula

$$
e(f)=e(n !)-e((l p) !)+e\left(f^{*}\right)
$$

given by Robinson, as a refinement of a formula offered formerly in NI.)

$(-1)^{q}$ is independent of the manner of taking $l p$-hooks from $T$. So, if $P$ is an element of $\Im_{n}$ having $l p$-cycles and $P_{0}$ is obtained from $P$ by removing those $l p$-cycles, as before, then

$$
\chi(P)=\chi(T ; P)=(-1)^{a} f^{*} \psi\left(T_{0} ; P_{0}\right)=(-1)^{a} f^{*} \gamma_{0}\left(P_{0}\right)
$$

where $\chi_{0}$ denotes the character belonging to $T_{0}$. If here $P$ has more than $l$ $p$-cycles, ${ }^{14)}$ then $\chi_{0}\left(P_{0}\right)=0$ whence $\chi(P)=0$ too. On the other hand, there exists certainly a (p-regular) element $P_{0}$ in $\Im_{m}$ with $\gamma_{0}\left(P_{0}\right) \neq 0$. If $P$ has exactly $l$ $p$-cycles, then $g(P) / g\left(P_{0}\right)=\frac{n !}{m ! l ! p^{l}}$ for the numbers $g(P), g\left(P_{0}\right)$ of conjugates. of $P, P_{0}$, in $\Im_{n}, \Im_{m}$ respectively. Hence, by $(8), f / f_{0}=\frac{f^{*} g(P)}{((p-1) !)^{l} g\left(P_{0}\right)}$ $\Pi\left(\widetilde{K}_{\lambda} \widetilde{L}_{\lambda}\right) \Pi M_{\lambda}$. Therefore $((p-1) !)^{l} \frac{g\left(P_{0}\right) \gamma_{n}\left(P_{0}\right)}{f_{0}}=\frac{f^{*} g(P) \chi_{n}\left(P_{0}\right)}{f} \Pi\left(\widetilde{K}_{\lambda} \widetilde{L}_{\lambda}\right)$ $\Pi M_{\lambda}$. Since $\Pi M_{\lambda} \equiv(-1)^{a}, \Pi\left(\widetilde{K}_{\lambda} \widetilde{L}_{\lambda}\right) \equiv 1 \bmod p$, we have, by (10),

$$
\frac{g(P) \chi(P)}{f}=\frac{g(P) \chi(T ; P)}{f(T)} \equiv \frac{g\left(P_{0}\right) \chi\left(T_{0} ; P_{0}\right)}{f\left(T_{0}\right)}(-1)^{l}
$$

14) Or, more generally, if $P_{0}$ has order divisible by $p$. 


$$
=\frac{g\left(P_{0}\right) \chi_{0}\left(P_{0}\right)}{f_{0}}(-1)^{\ell} \bmod p .
$$

This (11), generalization of (3), holds however also for $P$ with more than $l p$ cycles, since the both sides vanish then. Similarly as in $\S 1$ we obtain

II. Diagrams belonging to the characters of $\mathfrak{S}_{n}$ in a same p-block have a same p.core.

This II, counter-part of the latter half of the Toronto proof, may be combined with Brauer's exact estimate of the number of $p$-blocks in order to obtain a second proof of the theorem:

III. Two characters of $\mathfrak{S}_{n}$ belong to a same p-block if ond only if their diagrams have a same p-core.

In fact, it is one just a step to get III from II and the fundamental relation (11) by virtue of Brauer's theory of blocks. ${ }^{15)}$

Mathematical Institutes,

Nagoya University

Okayama University

15) The theory is summarized in Brauet, 1.c. 2). If $P^{\prime}$ is an element of $\mathfrak{S}_{n}$ possessing ip cycles of length 1 and $P_{0}$ is obtained from $P^{\prime}$ by removing those $l p$ cycles and if normalizer of $P_{0}$ in $\widetilde{G}_{m}$ has an order prime to $p$, then we can show from (11) $g\left(P^{\prime}\right) \chi\left(P^{\prime}\right) / f \equiv g\left(P_{0}\right) \chi_{0}\left(P_{0}\right) / f_{0} \bmod p$. 